\title{
Bingo et enjeux sociaux : l'exemple de la communauté urbaine de Seaside Tongoa à Port- Vila (Vanuatu)
}

\section{Alice Servy}

\section{(2) OpenEdition}

Journals

Édition électronique

URL : http://journals.openedition.org/jso/6502

DOI : $10.4000 /$ jso. 6502

ISSN : $1760-7256$

Éditeur

Société des océanistes

\section{Édition imprimée}

Date de publication : 15 décembre 2011

Pagination : 285-298

ISBN : 978-2-85430-037-4

ISSN : 0300-953x

Référence électronique

Alice Servy, «Bingo et enjeux sociaux : l'exemple de la communauté urbaine de Seaside Tongoa à Port-Vila (Vanuatu) », Journal de la Société des Océanistes [En ligne], 133 | 2e semestre 2011, mis en ligne le 31 décembre 2014, consulté le 30 avril 2019. URL : http://journals.openedition.org/jso/6502 ; DOI : $10.4000 /$ jso.6502 


\title{
Bingo et enjeux sociaux : l'exemple de la communauté urbaine de Seaside Tongoa à Port-Vila (Vanuatu)
}

par

\author{
Alice SERVY*
}

\section{RÉSUMÉ}

La pratique des jeux de hasard est interdite (sauf exceptions) au Vanuatu et est parfois affectée d'une forte charge négative. Deux formes d'un même jeu nommé bingo pratiqué avec des cartes à jouer se mettent en place dans la communauté urbaine de Seaside Tongoa à Port-Vila : l'une souvent considérée comme un simple "passe-temps", l'autre s'apparentant à une collecte de fonds. Nous verrons que ces jeux d'argent, outre leur dimension ludique et lucrative, laissent transparaître des enjeux sociaux. Si le bingo passe-temps alimente quotidiennement les sentiments d'appartenance aux ensembles résidentiels et aux groupes sexués, le bingo solidaire ou de collecte de fonds, localement appelé Katikati, renforce quant à lui des relations fondées sur la réciprocité des dons à une échelle plus large. Ces deux formes de bingo ne relèvent pas de logiques contradictoires. Elles contribuent à nourrir des sentiments d'appartenance communautaire multiples et multifactoriels qui peuvent être chacun vécus de manière intermittente.

Mots-Clés : Vanuatu, Port-Vila, bingo, jeux d'argent, enjeux sociaux

La législation de la République du Vanuatu définit le jeu de hasard comme " l'acte de parier, de jouer ou de faire jouer à un jeu comportant une mise ou un enjeu ». Cette pratique est interdite sur le territoire et dans les eaux de l'archipel en dehors des " cafés et hôtels patentés ou des pensions de famille à la condition toutefois que ces jeux ne revêtent pas le caractère de jeux de

\begin{abstract}
Gambling is prohibited (excepted in some cases) in Vanuatu and is sometimes affected by a strong negative charge. Two forms of gambling take place in the urban community of Seaside Tongoa in Port Vila: the first is seen as a daily pastime; the second is related to fundraising, solidarity and is occasional. The argument in this paper is that gambling, far from being only a gainful or leisure activity, reveals social issues. If the daily pastime bingo reinforces feelings of membership in residential settings and gendered social groups, occasional bingo named Katikati give rise to relationships based on reciprocity of gifts in larger wholes. Daily and occasional bingos are not in opposition. These two forms of gambling contribute to nourishing abundant and multifactorial community feelings each of which can be intermittently experienced.
\end{abstract}

Keywords: Vanuatu, Port-Vila, bingo, gambling, social issues

hasard publics " (Législation de la République du Vanuatu, 2006 : 2). Les jeux de hasard illégaux ne prennent à ma connaissance qu'une seule apparence dans la communauté urbanisée de Seaside Tongoa à Port-Vila, celle du bingo (loto). Mais ce jeu pratiqué avec des cartes à jouer y revêt une double forme. Au quotidien, le bingo est souvent qualifié de " passe-temps ", alors que de

\footnotetext{
* Doctorante à l'eness-CRedo à Marseille et au Bergen Pacific Studies Group (BPs Group) à Bergen (Norvège), alice. servy@free.fr
} 
façon plus occasionnelle, cette pratique s'apparente davantage à une collecte de fonds ou une action de bienfaisance. Le bingo ne constitue pas une activité exclusivement ni-vanuatu et est pratiqué dans des contextes (sociaux, géographiques, historiques, économiques, etc.) et sous des formes multiples (en matière de règles, de matériel et d'enjeux). En Nouvelle-Calédonie (province Nord), Éric Sabourin et Raymond Tyuienon (2007 : 301) ainsi que Denis Monnerie (2008: 41) rapportent l'existence de bingos associés à des marchés ruraux. Les premiers considèrent le bingo comme une transaction qui relève du " principe de réciprocité » et est " juxtaposée " aux échanges marchands réalisés lors de marchés de proximité (Sabourin et Tyuienon, 2007 : 302, 323). Le second décrit le bingo comme l'un des deux types d'échanges constituant le marché à caractère "redistributif " du village d'Arama. Adoptant une perspective plus large, il analyse ce jeu en relation avec d'autres systèmes d'échanges correspondant à diverses formes de sociabilité (façons d'être ensemble et d'interagir) et d'usages de la langue de la société contemporaine kanak (Monnerie, 2005 ; 2008). Dans l'esprit de l'analyse de Monnerie, nous allons ici tenter d'analyser les enjeux sociaux de la pratique de deux formes de bingo présentes dans la communauté de Seaside Tongoa. Tout en ayant un caractère à la fois ludique et lucratif, ce jeu de hasard contribue aussi à créer des liens sociaux solidaires et laisse transparaître l'existence de sentiments communautaires qui s'expriment à différents niveaux. Cette hypothèse s'insère dans une discussion plus générale sur le jeu institutionnalisé ainsi que dans une réflexion sur le concept de communauté.

\section{La communauté urbaine de Seaside Tongoa}

\section{Histoire de sa construction}

Dans les années 1920-1930, l'administration du Condominium franco-britannique régule de façon drastique la résidence des insulaires non originaires de l'île d'Efate - où se situe la capitale de l'archipel. Un migrant sans emploi ne peut demeurer plus de quinze jours à Port-Vila sans être menacé de retour forcé vers son île d'origine. À cette époque, la plupart des migrants signent des contrats à court terme qui leur garantissent un logement de fonction (Lightner et Naupa, 2005 : 51, 88). À partir de 1942, les activités non agricoles se développent et la mobilité circulaire - succession de déplacements de court terme, " répétitifs ou circulaires par nature " - s'intensifie à Port-Vila (Bedford, 1973 : 188, 202-203). Mais les migrants se considèrent toujours comme n'étant que de passage en ville et ont de ce fait peu d'exigences tant en matière de logement que de revenus (Bonnemaison, 1977 : 4). Ce n'est qu'à la suite de l'ouragan de 1951 qu'un grand nombre d'habitations " en dur" sont construites à Port-Vila (Lightner et Naupa, 2005 : 95). À partir des années 1960, des communautés urbaines voient progressivement le jour dans la

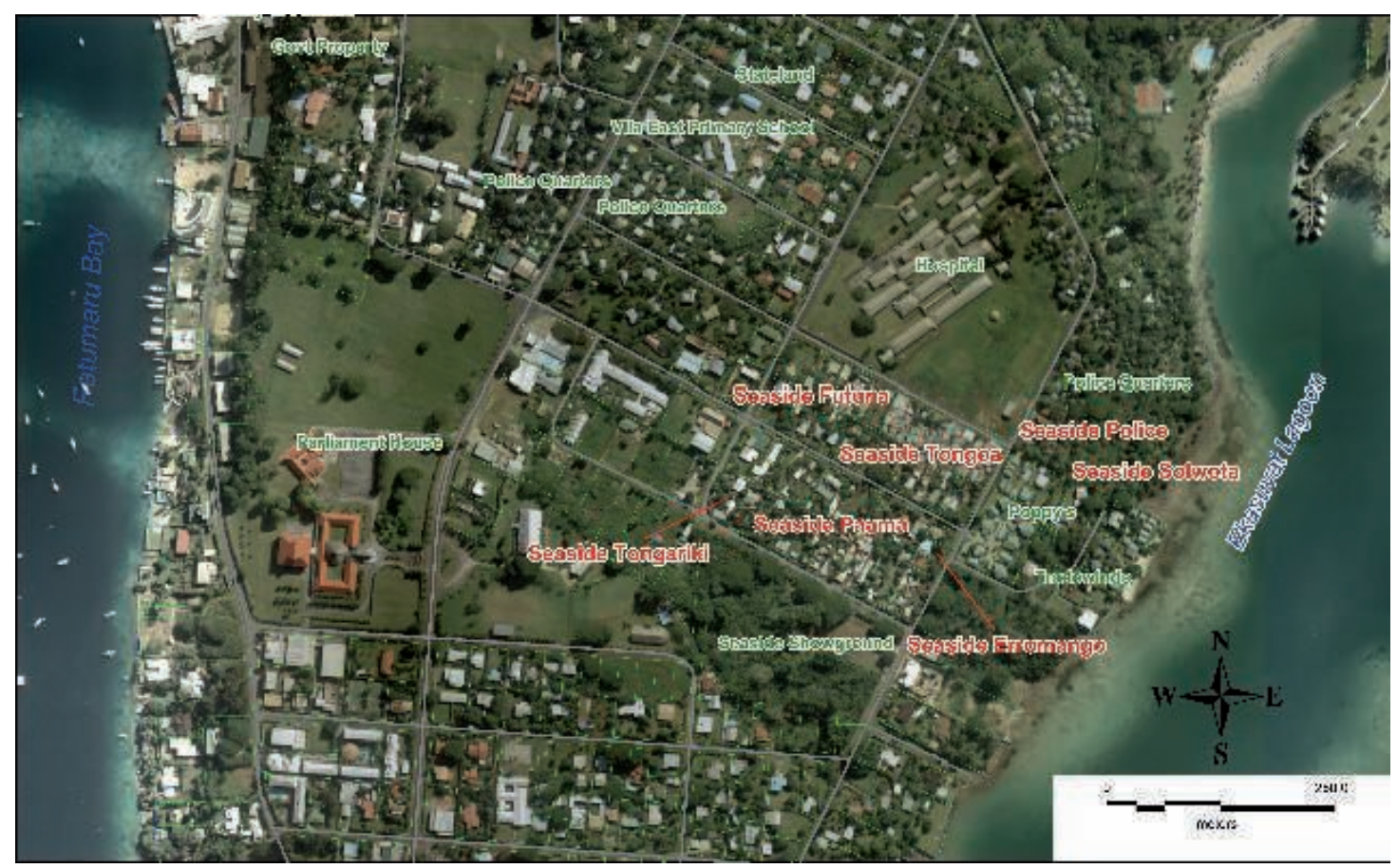

Carte 1. - Localisation de Seaside Tongoa à Port-Vila (C) the ITC Unit, Ministry of Lands and Natural Resources, 2011. We take no responsibility for the accuracy of information portrayed) 
capitale, suite au développement des secteurs d'activité économique et à l'augmentation des opportunités d'emplois à Port-Vila (Bonnemaison, 1977 : 4-6). Quelques familles de migrants venues principalement des îles Sheperd (Centre Vanuatu) s'installent en ville et forment des " extensions » communautaires fondées sur l'appartenance villageoise ou insulaire. Ces entités constituent dès lors des espaces d'hébergement pour les travailleurs temporaires engrangeant de faibles revenus (Charpentier, 1998 : 94 ; Bonnemaison, $1977: 6)$. Du fait de la mobilisation des réseaux de parenté lors de l'achat de parcelles privées ou communautaires, de nombreuses personnes y possèdent en effet un droit tacite de résidence (Bonnemaison, 1977 : 55).

Historiquement, la communauté de Seaside Tongoa s'est ainsi créée comme une cellule d'accueil et d'entraide pour les migrants résidant dans la capitale, comme un sas de communication entre le monde urbain et rural, permettant de se loger et de décrocher un travail. Des structures de régulation et des systèmes de solidarité parant aux aléas professionnels et financiers furent mis en place. Dès son acquisition, le terrain communautaire de Seaside Tongoa ainsi que ses parcelles privées constituèrent le lieu principal de rassemblement des personnes venues de Tongoa (la plus grande île des Sheperd) dispersées dans différents quartiers de Port-Vila (Bonnemaison, 1977 : 56-58). En 2009, la communauté comptait 700 personnes originaires dans leur grande majorité de cinq villages de l'île de Tongoa dont les membres parlent la langue namakura : Mangarisu, Bonga Bonga, Itakoma, Matangi, Euta et Purau ${ }^{1}$. Les habitants de cette communauté urbaine partagent une histoire, un lieu et mode de vie, une même origine insulaire et villageoise, des associations (religieuses, artistiques, sportives, de microcrédits), un système politique (chefferie), une culture et une langue - bien qu'ils parlent également bichlamar, langue véhiculaire du Vanuatu, ainsi que l'anglais ou le français lorsqu'ils ont été scolarisés.

\section{La dimension économique de la vie sociale}

Seaside Tongoa s'étend sur un espace d'environ $15000 \mathrm{~m}^{2}$ (carte 1) et est connu pour être l'un des quartiers les plus pauvres de la capitale. Dans les logements surpeuplés, principalement construits en tôle, cohabitent des migrants arrivés en ville il y a seulement quelques mois ou présents depuis plusieurs dizaines d'années, ainsi que leurs enfants voire petits et arrière-petits-enfants nés pour certains à Port-Vila. Ces personnes viennent chercher un emploi, visiter leurs proches, profiter des services de l'hôpital central (en particulier du service périnatal et de la maternité) ou souhaitent continuer leurs études dans la capitale. Peu d'habitants de Seaside Tongoa occupent un emploi salarié à temps complet, et rares sont les gens qualifiés et correctement rémunérés. Cependant, la grande majorité des personnes en âge de travailler mettent en place des activités lucratives plus ou moins pérennes et régulières (massages thérapeutiques ou petits commerces, par exemple) ou exercent un emploi salarié par intermittence. La place prise par l'argent dans la vie et les discours des personnes rencontrées est prépondérante. La plupart regrettent en effet la gratuité sublimée d'une vie au village. D'une voix quasi unanime, les habitants de Seaside Tongoa décrivent leur île comme une sorte de "paradis terrestre ", où tout serait gratuit. En fait, Susanna Katharine Kelly montre que l'argent et les façons de s'en procurer constituent l'une des principales préoccupations des habitants de l'île de Tongoa. Lécole, l'éclairage, les cérémonies, la santé et le transport des personnes et des marchandises vers la capitale constituent des postes de dépenses non négligeables en zone rurale (Kelly, 1999 : 80). La circulation d'argent entre les membres de la communauté urbaine de Seaside Tongoa est quotidienne : en particulier lors des collectes de fonds, des parties de bingo, des dons cérémoniels et des pratiques de soins traditionnels. Des relations de dettes et de dons monétaires ou matériels sont ainsi générées. Partager la nourriture est une valeur et une pratique fondamentale au Vanuatu. Lorsqu'un visiteur entre dans un foyer, il est d'usage de lui offrir à manger. Les actes de solidarité sont ainsi affichés, tandis qu'il est fait référence (en bichlamar) à la coutume de donner (givim) ainsi qu'aux devoirs chrétiens de charité (serem) et d'entraide (helpem) que la plupart des habitants de Seaside Tongoa respectent en tant que chrétiens. L'institution du don et sa triple obligation - donner, recevoir, rendre (Mauss, 1950) - ont fait l'objet de nombreux et remarquables travaux en Mélanésie. En 2010, l'un de mes amis de la communauté m’écrivait en français :

«Dans notre coutume, tout le monde travaille ensemble dans une vie commune où le partage règne sur le peuple indigène. " ${ }^{2}$

\section{Le sentiment d'appartenance communautaire}

Au Vanuatu, le sentiment d'appartenance nationale est quasi-inexistant (Tabani, 2002:254, 265). Les communautés insulaires et villageoises

1. Entretien du 27/08/2009 avec un homme d'une soixantaine d'années, elder de l'Église presbytérienne de Seaside Tongoa, ancien secrétaire du Paramount chief d'Itakoma.

2. Courrier électronique du 18/05/2010 à mon intention, envoyé par un homme d'environ 35 ans. 
ainsi que leurs multiples divisions et subdivisions se pensent et se donnent à voir sans que leur addition forme une nation. Le vocable communauté - komuniti en bichlamar - est fréquemment employé pour désigner des réalités multiples. En faisant appel à des auteurs classiques tels Tönnies, Durkheim ou Weber, nous définissons la communauté comme un agrégat social dont les membres partagent le sentiment d'appartenir à cet ensemble via la mise en exergue d'un certain nombre de traits distinctifs, déterminés et intériorisés au cours des générations (Leif, 1977 ; Durkheim, 1975, 2007 ; Weber, 1995). Ce ne sont pas des traits spécifiques qui font la communauté (territoire, langue, histoire, mode de recrutement), mais le fait de considérer ces caractéristiques comme des éléments distinctifs. Les sentiments communautaires sont abondants, d'origines multiples (pratique sportive, confession religieuse, etc.) et certains peuvent être éprouvés en certaines occasions seulement. Le sentiment d'appartenir à telle ou telle entité sociale est en effet alimenté par différentes façons d'interagir et d'être ensemble et ressenti avec plus ou moins de force (voire non éprouvé) en fonction des lieux et moments de sociabilité vécus par la personne (funérailles, parties de bingo, messe, marchés, etc.). Les communautés ne sont donc pas des entités figées, leurs frontières sont floues et mouvantes. Les habitants de Seaside Tongoa se pensent comme une communauté à la fois unie et parcellaire. Ainsi, chaque personne éprouve une multitude de sentiments d'affiliation communautaire. Chacun a conscience d'appartenir en même temps à un nasara - " groupe social originel " (Bonnemaison, $1970: 12$ ) - à un village, à une île, à un lieu d'habitation, à un groupe sexué, à une communauté religieuse, etc., mais chacune de ces appartenances pourra être vécue et sollicitée indépendamment des autres en fonction des circonstances. Les nombreux discours sur le partage que nous avons entendus et les modes d'usage de l'argent que nous avons observés nous permettent de dire que les formes de réciprocité, de don, de dette et d'entraide mises en place à Seaside Tongoa constituent des caractères que les habitants de cette entité mettent en avant pour se distinguer des autres communautés. Quatre domaines où il est fait usage d'argent ont été étudiés lors de mon terrain de recherche à Seaside Tongoa : les dons cérémoniels, les services, les jeux de hasard et les dépenses quotidiennes, en particulier d'ordre alimentaire. Cet article s'attache tout particulièrement à détailler des faits économiques laissés pour compte dans les études anthropologiques ${ }^{3}$ menées à Port-Vila : les jeux d'argent et leurs enjeux sociaux.

\section{Principes et histoire du bingo}

\section{Le bingo comme forme spécifique du loto}

Le bingo est un jeu de hasard très populaire en Europe, connu en France sous le nom de loto. Ce terme d'origine anglaise signifie en effet " loto " ou " loterie " (Sabourin et Tyuienon, 2007 : 315). En France, mais aussi dans le Pacifique - comme par exemple à Arama, en NouvelleCalédonie (Monnerie, 2008 : 38) -, le bingo se joue avec des cartons portant quinze chiffres répartis sur trois lignes horizontales. Le meneur de jeu tire à l'aveugle des jetons sur lesquels sont inscrits des numéros et les annonce aux participants. L'objectif de chacun des joueurs est d'être le premier à avoir les cinq numéros d'une même ligne tirés (une quine) ou mieux les quinze numéros d'un même carton (un bingo). Le gagnant remporte des lots (biens ou services) ou une partie de l'argent collecté grâce à la location des cartons. Dans les formes de jeux de tirage (loterie) les plus modernes, pratiquées à une échelle nationale ou transnationale telles que le Loto, le Joker+ et le Keno de la Française des jeux ou l'Euro Millions, l'enregistrement des chiffres choisis (ou non) par les joueurs est informatisé. Le tirage des numéros se fait à l'aide d'appareils électroniques et est annoncé à travers les médias afin que le(s) gagnant(s) récupère(nt) ses(leurs) gains.

Le bingo pratiqué à Seaside Tongoa en 2009 est une forme spécifique du loto qui utilise des cartes à jouer à la place des cartons et jetons à numéros. L'objectif de chaque joueur de bingo est que les cartes annoncées par le meneur de jeu correspondent aux cartes qu'il a en sa possession. Plusieurs paquets de 34 cartes (32 cartes plus 2 jokers) sont nécessaires en plus du paquet complet utilisé pour le tirage. Chaque joueur paie généralement 10 ou 20 vatus $(0,07$ ou $0,14 €)$ pour obtenir cinq cartes d'un même paquet. S'il souhaite posséder plusieurs sets de cinq cartes afin d'augmenter sa probabilité de gagner, il lui suffit de payer en fonction du nombre de sets voulu. Dans la forme classique du loto, un set de cinq cartes correspondrait à l'une des lignes à cinq numéros d'un carton. Au début de chaque manche de bingo, le paquet de tirage est battu et les deux dernières cartes de ce tas sont révélées et exclues du jeu. Les personnes ayant l'une ou les deux de ces cartes en main les retirent et les remplacent par de nouvelles cartes. Le gagnant

3. Robert Rubinstein a publié un article sur un jeu nommé «Last Card " pratiqué dans les années 1970 sur l'île de Malo (Vanuatu). Mais cette activité comportait rarement une mise en jeu d'argent. La pratique de Last Card reflétait selon l'auteur les tensions politiques et foncières précédant l'indépendance du Vanuatu (1987). 
de la dernière manche tire les cartes une à une dans le paquet complet tenu face cachée, les annonce en bichlamar ${ }^{4}$ à l'assemblée et les pose face à lui. Certaines personnes, en particulier les femmes âgées, peuvent se faire remplacer lorsque c'est à leur tour de tirer et d'annoncer les cartes aux autres joueurs. Un débit de parole rapide est en effet nécessaire, ou du moins souhaité par les joueurs de Seaside Tongoa. Lorsqu'un participant entend que l'une de ses cartes est annoncée, il la cache ou la retourne. Le premier joueur dont les cinq cartes du même set ont été tirées crie « bingo » et remporte la mise collectée une fois sa victoire confirmée. Chacun doit donc décider du nombre de sets joués (c'est-à-dire du montant engagé dans chaque partie) et savoir s'arrêter avant d'avoir dépensé tout son argent.

\section{Un phénomène récent}

Peu de données historiques sur la naissance ou le développement des jeux de hasard dans l'archipel mélanésien du Vanuatu sont en notre possession. Mais des travaux menés ailleurs en Mélanésie laissent supposer que les jeux de hasard ont fait leur apparition au Vanuatu suite aux premiers contacts avec l'Occident (et ses monnaies) et se sont développés sous la colonisation (Mitchell, $1988: 643$; Hayano, 1989 : 231 ; Zimmer, 1987 : 22). C'est le cas en NouvelleCalédonie où le bingo a été introduit en milieu kanak par les missions protestantes puis catholiques, avant d'être utilisé par les associations pour recueillir des fonds (Sabourin et Tyuienon, 2007 : 315). Les dominos, les jeux de dés ou de cartes sans mise ou enjeu sont pratiqués à PortVila depuis plusieurs décennies, mais, d'après un groupe de joueuses régulières ${ }^{5}$, la pratique des jeux d'argent à Seaside Tongoa est un phénomène récent, importé de Nouvelle-Calédonie. Deux habitantes de cette communauté urbaine seraient ainsi revenues de Nouméa aux alentours de 2005 avec des cartons de loto. Puis du fait de l'engouement des femmes, de la rareté de ces cartons et de leur coût, elles auraient commencé à utiliser des cartes à jouer après qu'une parente habitant dans un quartier voisin fut venue en 2008 leur enseigner cette pratique. Selon une ancienne employée de maison ${ }^{6}$, un groupe d'une douzaine de femmes australiennes et néozélandaises vivant à Port-Vila jouent au bingo avec des cartes à jouer depuis plusieurs années. Elles se réunissent chez l'une d'entre elles, les vendredis après-midi de jour de paye, et misent 3000 vatus $(20 €)$ par manche de douze points. La première ayant réalisé douze bingos remporte les gains. Seaside Tongoa n'est donc pas la première communauté mélanésienne de Port-Vila à avoir joué au bingo, mais cette activité semble y avoir pris là-bas une ampleur considérable. Estce parce que les habitants de Seaside Tongoa, dont beaucoup ne possèdent pas d'emploi salarié à temps complet, disposent de longues heures à occuper quotidiennement ou bien, comme le suggère Monnerie, parce qu' " ajouter des systèmes d'échanges est une façon dont les sociétés se renouvellent et se renforcent " (Monnerie, $2008: 28)$ ?

\section{Un loisir communautaire au quotidien ?}

\section{Une communauté résidentielle de joueuses}

Les parties de bingo quotidiennes sont organisées de façon informelle à Seaside Tongoa. Mais une certaine régularité est observable. En 2009, nous avons pu ainsi distinguer quatre ou cinq lieux où des joueurs se rassemblaient quotidiennement. Les parties se mettent généralement en place après le déjeuner, une fois visionné le dernier épisode de la sitcom latino-américaine du midi. Selon une habitante protestante de la localité, il ne faut pas jouer au bingo le dimanche "parce que c'est le jour du seigneur ". Cependant, d'après mes observations, des parties de bingo sont disputées quotidiennement au sein de petits groupes de joueurs bien qu'ils soient eux aussi protestants. Ces groupes de quatre à vingt personnes sont constitués de voisins ou de parents (consanguins et affins résidant localement). Le noyau fixe est généralement formé de femmes habitant sur une même parcelle de terrain, rejointes à l'occasion par des membres de leur famille respective. À Seaside Tongoa comme dans la province Nord de Nouvelle-Calédonie (Sabourin et Tyuienon, 2007 ; Monnerie, 2008), la communauté de joueurs est donc avant tout résidentielle et essentiellement de sexe féminin. De jeunes adolescentes et des hommes se joignent parfois aux groupes de femmes. Mais ils ne restent que pour quelques manches tandis que les joueuses peuvent participer pendant plusieurs heures. Les jeux d'argent étudiés en Mélanésie dans les années 1970-1980 - principalement pratiqués avec des cartes à jouer - étaient soit

4. Les figures des cartes sont nommées en bichlamar: las (as), king (roi), misis (dame), jek (valet), ten (dix), naen (neuf), eit (huit), seven (sept) et joka (joker). Les couleurs sont appelées : pik (pique), club (trèfle), tiaman (carreau), hat (cœur), red (rouge), blak (noir). La figure est annoncée avant la couleur de la carte. L'as de carreau est ainsi nommé las tiaman. Les termes red et blak sont parfois employés seuls pour désigner les jokers rouge et noir.

5. Discussion du 11/06/2011 avec des joueuses de Seaside Tongoa âgées d'une quinzaine à une cinquantaine d'années.

6. Entretien du 02/07/2011 avec une habitante de Seaside Tongoa âgée de quatre-vingts ans, employée de maison à la retraite depuis 2009 dont l'ancienne patronne organise chez elle des parties de bingo. 
mixtes tels que le Lucky chez les Daulo (Sexton, 1987) et le Trip-lip chez les Gende (Zimmer, 1986) de Papouasie Nouvelle-Guinée, soit plutôt masculins comme le Satu (jeu de dés) joué par les Wape (Mitchell, 1988), le Last Card par les Gende (Zimmer, 1987) et le Trip-lip par les Awa (Hayano, 1989) en Papouasie NouvelleGuinée ainsi que le Last Card pratiqué sur l'île de Malo au Vanuatu (Rubinstein, 1987). Ces parties de cartes ou de dés non mixtes exprimaient une différence et une séparation des mondes sociaux féminins et masculins. Contrairement aux hommes travaillant à Port-Moresby et dans les plantations du pays, les femmes awa disposaient ainsi d'un accès limité à l'argent dans les années 1970-1980 et n'avaient pas la possibilité d'apprendre à jouer aux cartes (Hayano, 1989 : 234236). Chez les Tiwi du Nord de l'Australie, si les personnes de l'un et l'autre sexe prenaient part aux jeux d'argent, les formes engageant d'importantes sommes étaient généralement pratiquées par les hommes qui, en cas de victoire, redistribuaient leurs gains de façon à maintenir un large réseau social. Quant aux parties mettant en jeu de plus faibles mises, elles permettaient aux femmes de faire circuler l'argent disponible entre leurs foyers afin d'acheter des produits de première nécessité (Goodale, 1987 : 19). À Seaside Tongoa en 2009, si le bingo laisse transparaitre une différence entre les mondes sociaux masculins et féminins, ce ne sont pas les femmes qui sont mises (ou se mettent) à l'écart de ce jeu d'argent mais les hommes qui ne daignent pas y participer. Les parties quotidiennes de bingo forment ainsi de petites communautés résidentielles et féminines. Loin de n'être qu'un simple loisir ou passe-temps, cette activité nourrit jour après jour des sentiments d'appartenance à des ensembles sociaux localisés et sexués.

\section{Un jeu prohibé et mal considéré}

D'après Paul Yonnet, « le loisir est une quantité de temps libre affranchi des exigences du temps obligé (celui du travail professionnel ou scolaire et des astreintes qui s'y attachent : transport, etc.) et du temps contraint (celui des obligations sociales, administratives, familiales et domestiques)» (1985 : 64). Si le loisir paraît être une activité « libre », il n’est cependant jamais dégagé des obligations et contraintes sociales, culturelles, matérielles, économiques et politiques. Ainsi, pratiquer un sport (golf ou football, par exemple) ou participer aux parties de bingo à Seaside Tongoa relèvent de la socialisation et de pressions extérieures (celles d'un groupe de femmes ou de l'époux, par exemple), plus que du choix d'un individu autonome et fictif qui serait dépourvu d'un environnement social. Qualifier le bingo pratiqué quotidiennement à Seaside Tongoa de loisir, au sens où l'entend Yonnet, est ainsi profondément réducteur mais fait écho à la charge négative que certains lui affectent localement. Les hommes interrogés à Seaside Tongoa - bien souvent, des conjoints de joueuses - jugent ainsi le bingo peu intéressant et considèrent qu'il engendre des pertes d'argent. D'après une habitante de la communauté voisine de Seaside Paama (carte 1), un certain nombre d'époux pensent que le bingo "gâche " les vatus qui leur auraient servi à payer du kava - boisson narcotique confectionnée à partir des racines du poivrier sauvage - ou des cigarettes. Certains man Tongoa - nom donné aux personnes originaires de l'île de Tongoa ou y habitant - pensent quant à eux que ce jeu ne leur permet pas de gagner d'assez grosses sommes, contrairement aux machines à sous ou au poker. Au bingo, chaque participant dépense entre 100 et 500 vatus $(0,68$ et $3 €)$ par séance en ayant en moyenne deux sets de cinq cartes en main. Chaque gagnant perçoit entre 300 et 390 vatus ( 2 et $3 €$ ) par manche en fonction du nombre de joueurs et de sets joués par chacun. Au Club Vanuatu $^{7}$ et dans le casino de l'hôtel Le Sebel ${ }^{8}$, les joueurs observés misent environ 1000 vatus $(7 €)$ et peuvent gagner plusieurs dizaines de milliers de vatus. En août 2009, une habitante de Seaside Police (carte 1) d'une cinquantaine d'années a ainsi touché 45000 vatus (306 €) aux machines à sous, soit presque deux fois le salaire mensuel minimum légal pour un emploi à temps complet $^{9}$ au Vanuatu. Les femmes de Seaside Tongoa se rendent rarement dans les casinos car davantage d'argent est en jeu ${ }^{10}$. Cependant, lors d'observations au Club Vanuatu et à l'hôtel Le Sebel, trois fois plus de femmes que d'hommes ont été comptées. La plupart des hommes interrogés à Seaside Tongoa reprochaient aux joueuses d'être " paresseuses " et de mal s'occuper de la maison

7. Observation du 14/08/2009 aux alentours de $18 \mathrm{~h}$ au Club Vanuatu : trois hommes et dix femmes jouant aux machines à sous ont été dénombrés. Les trois hommes étaient regroupés au fond de la pièce, côté droit. Tous les joueurs étaient ni-vanuatu.

8. Observation du 10/08/2009 au casino de l'hôtel Le Sebel en compagnie de deux amies d'une cinquantaine d'années, l'une habitant à Seaside Police et originaire de l'île de Nguna, l'autre à Seaside Tongoa. La plupart des joueurs étaient nivanuatu, mais une poignée d'expatriés et de touristes se trouvaient également dans le casino de l'hôtel. Parmi la trentaine de personnes regroupées devant les machines à sous, environ deux tiers étaient des femmes. Les hommes s'étaient plutôt rassemblés au fond du casino tandis que les femmes se situaient vers la porte d'entrée.

9. Le salaire mensuel minimum légal pour un emploi à temps complet est de 26000 vatus (177 €) pour $8 \mathrm{~h} /$ jour, $44 \mathrm{~h} /$ semaine, 22 jours/mois.

10. Discussion du 14/10/2009 et 28/10/2009 avec des joueuses d'une trentaine et cinquantaine d'années. 
et des enfants : plats mal équilibrés, manque de pédagogie, défaut de surveillance, etc. Ces réprimandes seraient également monnaie courante en Nouvelle-Calédonie. Ainsi, d'après l'article paru dans Les Nouvelles calédoniennes repris dans le Vanuatu Infos du vendredi 28 août 2009, on reproche aux femmes de Nouméa jouant au bingo de ne pas surveiller les devoirs de leurs enfants (Anonyme, 2009). Selon certains man Tongoa:

"Les joueuses n'ont pas conscience des difficultés de la vie car elles vivent sur une terre communautaire et ne paient pas de loyer. Elles gâchent leurs journées au lieu de chercher un travail."

Les femmes qui ne jouent pas ou qui n'habitent pas à Seaside Tongoa critiquent parfois elles aussi ces joueuses qui ne " nettoient pas la maison ", " ne veulent pas faire à manger ", etc. Selon une habitante de la communauté ${ }^{11}$, certaines personnes vont voir la police pour tenter d'empêcher les femmes de jouer au bingo. Elles cherchent à ce qu'un "appareil adéquat " fasse appliquer la loi (Becker, 1985 : 172). Quelques " animateurs de croisade morale " existent ainsi à Seaside Tongoa, mais leurs motivations ne sont pas humanitaires. Les "dénonciateurs" cherchent davantage à servir leurs intérêts, en obligeant leur épouse ou leur sœur à passer davantage de temps à s'occuper du foyer et des enfants (Becker, 1985 : 172, 183). Bien que ce jeu soit illégal, les agents de police répondent à ces " entrepreneurs de morale " qu'ils ne peuvent rien y faire. D'après le discours officieux de deux gradés ${ }^{12}$, personne ne juge nécessaire d'intervenir auprès des joueurs. La police a connaissance des lieux de délit : des parties seraient ainsi organisées par des chauffeurs de taxi et de bus attendant les clients devant les hôtels et des groupes de joueurs se réuniraient en fin d'aprèsmidi en centre-ville (à Seafront) et dans le quartier de Freswota. Mais en raison d'un manque de moyens, les représentants de la loi disposent d'un "grand pouvoir d'appréciation " sur ce qui doit être traité en priorité (Becker, 1985 : 183). Étant donné que la norme n’est pas appliquée ni cette pratique collective sanctionnée, celle-ci ne semble pas réellement créer une catégorie de déviants (Becker, 1985 : 186-187). La déviance est, selon Howard S. Becker, " une action publiquement disqualifiée " $(1985: 186)$. Mais seule une partie des hommes et des femmes de Seaside Tongoa dévalorise réellement cette pratique. Les remontrances vis-à-vis des joueuses sont principalement d'ordre privé. Ainsi, bien que n'ayant pas pu recueillir de témoignages précis sur le sujet, la violence domestique verbale et physique serait parfois conséquente aux parties de cartes. Du fait des remarques de leur mari, il arrive que des femmes ${ }^{13}$ jouent en cachette lorsque celuici est au travail. Certains hommes ${ }^{14}$ pensent au contraire que leurs femmes ont le droit de jouer leurs vatus si elles le souhaitent, même s'ils considèrent qu'elles perdent l'argent qui leur aurait permis d'acheter à manger ou du kava.

Lors des messes protestantes tenues à Seaside Tongoa en ma présence, le thème des jeux d'argent (gambling) n’a pas été abordé. Néanmoins, la branche ni-vanuatu de la Ligue pour la Lecture de la Bible (Scripture Union Vanuatu) a créé un livret dénonçant les dangers de ce type d'activités - qu'elles soient exercées au sein du casino, de la communauté ou de la famille (Paschke, 2008). Jouer est donc globalement mal considéré sauf lorsque cette activité est un prétexte pour ramasser des fonds (fundraising, voir plus loin).

\section{Des motivations pécuniaire et sociale}

Les femmes interrogées expriment leur motivation pour pratiquer quotidiennement le bingo en termes de gain d'argent. L'une des joueuses mannonce ainsi empocher parfois jusqu'à 1000 vatus $(7 €)^{15}$. Cependant, lorsque je lui demande combien elle gagne mensuellement grâce au bingo, elle me répond participer pour passer le temps. Une femme ${ }^{16}$ venue à Port-Vila pour accoucher m'explique qu'elle joue également à Tongoa le dimanche ou le soir en rentrant du jardin, mais que depuis qu'elle se trouve en ville, sa pratique est devenue quotidienne parce qu'elle n’a "rien à faire" : pas de jardins, de vaches ni de cochons dont s'occuper. Si rares que soient les femmes de la communauté ne participant pas aux parties de bingo, les joueuses ont tendance à ne pas avoir d'emploi fixe - bien que certaines salariées jouent le week-end ou en rentrant du travail. La volonté de gagner de l'argent et de passer le temps est donc ici soulignée.

Chez les Awa de Papouasie Nouvelle-Guinée, l'argent gagné et perdu aux cartes circulait de façon imprévisible et erratique parmi un groupe tournant de travailleurs migrants (Hayano, 1989 : 241). De la même façon, à Seaside Tongoa, une joueuse ${ }^{17}$ explique que " des fois tu

11. Entretien du 11/10/2009 avec une joueuse de Seaside Tongoa d'une cinquantaine d'années.

12. Ces deux policiers habitent à Seaside Police (à quelques mètres de Seaside Tongoa). Cette discussion autour des jeux d'argent à Vanuatu eut lieu le 09/10/2009.

13. Entretien du 11/10/2009 avec une habitante de Seaside Tongoa âgée d'une cinquantaine d'années.

14. Discussions des 14, 20 et 21/10/2009 avec deux hommes d'une trentaine d'années et une jeune femme de vingt ans.

15. Entretien du 14/10/2009 avec une joueuse d'une cinquantaine d'années.

16. Entretien du 11/10/2009 avec une femme d'une quarantaine d'années.

17. Entretien du 11/10/2009 avec une joueuse d'une trentaine d'années. 
gagnes, des fois tu perds. Une femme gagne un jour, le lendemain c'est une autre ». Sur le long terme, la redistribution globale de l'argent ne crée pas de différence perceptible de richesse ou de privilèges au sein de la communauté de Seaside Tongoa, comme au sein du village des Awa (Hayano, 1989 : 241). Aucune joueuse ne déclare gagner de façon continuelle. Chez les Wape de Papouasie Nouvelle-Guinée, si un homme gagne aux dés, il y a de fortes sanctions verbales s'il décide de quitter le jeu (Mitchell, 1988 : 643). S'il doit partir, on attend de lui qu'il rejoue le lendemain afin de laisser aux perdants une chance de recouvrer leurs mises (Mitchell, 1988 : 643). Le gagnant peut réserver une partie de ses gains pour aider à répondre à une obligation d'échange entre parents, tandis que le reste est dépensé pour des objets de première nécessité comme des habits pour la famille, des piles pour la lampe électrique ou pour jouer à la prochaine partie de dés (Mitchell, 1988 : 643). Chez les Awa, il existe de fortes pressions pour que les gagnants prêtent immédiatement leurs gains aux perdants. De même, s’il est temps de dépenser l'argent gagné dans du poisson en conserve et du riz, la nourriture doit être partagée (Hayano, 1989 : 242). À Seaside Tongoa, les joueuses de bingo déclarent pouvoir s'arrêter après avoir gagné ou perdu leur mise. Cependant, selon mes observations, les femmes quittent le plus souvent la partie lorsqu'elles ont perdu la somme qu'elles s'étaient fixé de jouer. Un certain nombre d'entre elles retournent malgré tout chercher de la monnaie chez elle et recommencent à jouer. D'après ce que nous avons pu voir, cet argent est utilisé pour acheter des friandises aux enfants - une glace à l'eau fabriquée par une des voisines à 10 vatus $(0,07 €)$ ou des chips au magasin asiatique à $20 / 30$ vatus $(0,14 / 0,20 €)$ - ainsi que le kava ou le repas du soir (riz, thon, pain, etc.). Tout comme chez les Awa, les flux monétaires résultant des parties de bingo ne suivent pas les chaînes traditionnelles de la parenté et du pouvoir (Hayano, 1989: 241). Cependant, à Seaside Tongoa, l'assemblée de joueuses paraît plus enjouée lorsque c'est une personne âgée qui gagne la partie que dans tout autre cas. Les femmes aident également les doyennes en vérifiant qu'elles ont bien retourné les cartes qui ont été annoncées. Et ceci est encore plus vrai lors des parties de bingo de collecte de fonds que nous allons présenter maintenant.
Bien que les parties quotidiennes de bingo soient globalement mal considérées et que les femmes affichent un dessein ludique et lucratif, des pressions sont simultanément exercées sur les simples observatrices. Ainsi, des reproches sont adressés aux femmes ne daignant pas prendre part au jeu. Ces remarques révèlent l'importance sociale que revêt cette pratique. Le bingo au quotidien souligne l'existence de communautés fondées sur le genre, le lieu de résidence et le village d'origine (chaque petit groupe rassemble en effet voisines et parentes) et contribue à entretenir des liens sociaux communautaires grâce au partage d'une activité qui ne peut donc pas simplement être qualifiée de loisir.

\section{Une forme spécifique d'échange redistributif}

\section{Activité mixte sans but lucratif}

La seconde forme de bingo pratiquée à Seaside Tongoa, appelée en bichlamar Katikati, peut être nommée bingo solidaire ou de collecte de fonds et s'apparente aux bingos dominicaux des marchés d'Arama (Nouvelle-Calédonie) décrits par Monnerie (2008 : 38). À Seaside Tongoa, les parties de cartes sont généralement mises en place les vendredis soir de jour de paie ${ }^{18}$ par une personne souhaitant récolter de l'argent pour une occasion particulière, par exemple régler les frais de scolarité de ses enfants, ou pour ses dépenses usuelles. À Arama, les bingos joués avec des cartons sont quant à eux organisés par des groupes de personnes vivant sur un même district ou par des associations (souvent liées à l'Église catholique) ayant besoin d'argent pour une raison bien définie et implicitement reconnue légitime du fait de son utilité pour la communauté. L'organisateur du bingo de Seaside Tongoa achète des lots ${ }^{19}$ d'une valeur comprise entre 70 et 200 vatus $(0,48$ et $1 €)$ et tout l'argent mis en jeu lors des parties lui revient. Les lots proposés concernent la cuisine et à l'hygiène quotidienne, domaines généralement féminins. En effet, s'il arrive à quelques hommes de Seaside Tongoa de faire la cuisine, l'hygiène (linge, vaisselle, sol) est presque toujours une affaire de femmes. Chaque gagnant choisit son bien. Rien n'est pris en double. Mais contraire-

18. J'ai assisté à deux bingos solidaires : le premier entre 20 h et 22 h 30, le 15/10/2009 et le second entre 19 h 30 et 21 h 30 le 23/10/2009. Au Vanuatu, la plupart des salariés reçoivent leur paye toutes les deux semaines, soit un vendredi sur deux.

19. Lors de la séance du 15/10/2009, il y avait entre 48 et 50 lots mis en jeu : 4 sachets de Milo (l'équivalent de notre Ovomaltine) avec un petit pot de beurre de cacahuètes par sachet, 4 boîtes de sauce tomate, 4 rouleaux de papier toilette avec un savon par rouleau, 4 bouteilles de jus de fruit concentré à diluer de $500 \mathrm{ml}, 4$ sachets de $1 \mathrm{~kg}$ de sucre de canne, 4 boîtes de sel de table, 4 tasses, 4 paquets d'épingles à linge en bois, 4 sachets de lessive, 4 sachets de biscuits avec un savon par sachet, 8 ou 10 paquets de biscuits de trois marques différentes. Lors de la séance du 23/10/2009, il y avait 38 lots mis en jeu : 2 bassines, 5 bouteilles de jus de fruit concentré, 4 tasses, 4 paquets de sel de table, 4 sachets de $1 \mathrm{~kg}$ de sucre de canne, 4 sachets de lessive, 8 paquets de gâteaux de trois marques différentes, 2 couteaux de cuisine, 5 flacons de parfum pour femme (de contrefaçon). 
ment à ce que l'on aurait pu imaginer, les premiers lots à partir ne sont pas les plus onéreux. Ainsi, une joueuse prend le papier toilette et le savon à 70 vatus $(0,48 €)$ alors qu'il reste encore un sachet de sucre à 200 vatus $(1 €)$. Dans une optique économique, les choix des participants apparaissent ainsi peu rationnels. Les gagnants prennent simplement ce dont ils ont besoin chez eux à court terme, semblant même parfois préparer leur petit-déjeuner du lendemain matin. Toutes les femmes, à une exception près, ont choisi du beurre de cacahuètes après avoir gagné un paquet de biscuits. Sauf pour l'organisateur, les parties de bingo solidaire n'ont pas de but lucratif et les joueurs ne cherchent pas à maximiser leurs profits matériels. À Seaside Tongoa, de la même façon que la majorité des dons des cérémonies nuptiales ou funéraires sont rarement conservés pour un prochain échange (les robes sont portées, les nattes étalées sur le sol), les marchandises du bingo sont consommées aussitôt. Les lots sélectionnés pour le bingo diffèrent cependant profondément des objets cérémoniels (nattes, nourritures cuites ou crues). Seule la présence de l'argent liquide se retrouve dans ces deux activités d'échanges.

Si les hommes sont nettement plus nombreux lors des parties de bingo solidaires que lors des séances quotidiennes ${ }^{20}$, la présence féminine est toujours écrasante. Mais le discours des hommes sur cette forme de bingo change du tout au tout. Alors que les critiques sur le bingo passe-temps sont vives, celles sur le bingo de collecte de fonds sont positives. Lors des deux soirées observées à Seaside Tongoa, non seulement les détracteurs du premier considèrent que le jeu a cette fois été organisé pour soutenir une cause, mais ils y participent eux-mêmes de bon cœur. De façon générale, les personnes qui ne jouent jamais aux bingos solidaires sont considérées comme égoïstes. Ces bingos attirent beaucoup de monde - entre 30 et 50 participants contre de 4 à 20 lors des jeux quotidiens. Enfin, les joueurs n'habitent pas tous dans la communauté de Seaside et ne sont pas tous des man Tongoa bien que la grande majorité soit originaire de cette île. Ces caractéristiques varient en fonction des soirées de jeu. La première séance de bingo solidaire observée dans la communauté attira ainsi un plus grand nombre de personnes, qui dépensèrent davantage d'argent que lors de la seconde soirée. Tout comme les petits et grands marchés d'Arama (NouvelleCalédonie) décrits par Monnerie (2005 : 129; 2008 : 40), les bingos du quotidien et ceux organisés lors d'occasions exceptionnelles mettent en jeu des groupes différents.

\section{Solidarité économique à tour de rôle}

D'après mes calculs, entre 5000 et 5500 vatus (34 et $37 €$ ) furent dépensés par les organisateurs en amont de chaque séance. Lors du premier bingo solidaire orchestré par une femme de Seaside Tongoa, tout en dépensant moins d'argent pour l'achat des lots, celle-ci a mis plus en jeu que l'organisatrice du second bingo et collecté 25650 vatus $(174 €$ ) contre 10940 vatus $(74 €)$ pour la seconde. En effet, la première organisatrice, sa cousine et un chef de la communauté voisine de Seaside Futuna (carte 1) constituaient un trio d'entraide. Chacun d'entre eux organise un bingo de collecte de fonds une fois par mois. Avant de commencer et afin de pouvoir jouer sans redonner à chaque manche de la monnaie, les trois associés mettent 3000 vatus $(20 €)$ par personne dans la boîte destinée à l'organisatrice. A la fin de la partie que j'ai observée, le chef de Seaside Futuna déclara publiquement avoir dépensé au moins 10000 vatus (68€) dans la soirée. Il demanda à l'organisatrice de remercier les autres joueurs venus les rejoindre et annonça la date de son prochain bingo et son attente d'une aide équivalente en retour. À l'inverse, l'organisatrice du second bingo solidaire n'était pas membre d'une " association ». Elle ne participa pas aux différentes manches - parce qu'elle s'occupait de la vente de plats - et ne prit pas la parole pour remercier les joueurs à la fin de la soirée. Une solidarité inter-résidentielle à l'échelle de Seaside Tongoa (terrain communautaire et parcelles privées), des différents groupements de man Tongoa de Port-Vila et du quartier de Seaside (en l'occurrence entre Seaside Futuna et Seaside Tongoa) se met donc en place par l'intermédiaire des bingos solidaires et gagne en force au sein de petits groupes organisés tels que la triade précédemment décrite. Cette aide économique est prise en charge à tour de rôle, non seulement par les membres de cette triade, mais aussi par les différents participants des bingos solidaires. $\mathrm{Si}$ un tour de rôle ne semble pas être clairement établi, comme à Arama (Monnerie, 2005 : 127), chaque personne souhaitant organiser un bingo ou une collecte d'argent sera néanmoins soutenue par ceux qu'elle a précédemment aidés à rassembler des fonds. Les soirées de bingo solidaire constituent ainsi une forme d'échange redistributif apparentée au fundraising.

\section{Bingo et fundraising}

De même que les dons cérémoniels à Seaside Tongoa ne sont pas faits dans le but d'enrichir les familles, les bingos relèvent davantage de l'enquotidiens, on le sait, les hommes se font très rares et, le plus souvent, les parties sont exclusivement féminines. 
traide, de la réciprocité que de l'échange marchand. Le bingo solidaire s'apparente ainsi fortement aux collectes de fonds appelées fundraising. Ces collectes prennent une grande ampleur au Vanuatu, en particulier à Port-Vila. Les fundraising constituent une activité sociale et économique régulière. Plusieurs raisons sont généralement avancées pour organiser une telle collecte : régler les frais de scolarité, payer le loyer, financer un voyage (dans l'archipel ou à l'étranger), améliorer le quotidien de la famille, organiser un mariage, alimenter les caisses d'une association, d'une Église, etc. Annelin Eriksen note ainsi, qu'en 1999, à Ranon (Ambrym), des fundraising de différents types sont mis en place de façon très régulière au profit de l'Église presbytérienne du village. Les femmes organisent ainsi des petits marchés ou des ventes de plus grande envergure $^{21}$ (2008 : 99-103). En dehors de ces collectes de fonds (qui n'ont pu être observées à Seaside Tongoa), les fidèles donnent également mensuellement ou bimensuellement de l'argent (ou des biens). Les fundraising, les bingos solidaires ou les dons cérémoniels fonctionnent sur le même principe. Ainsi, tout comme l'organisateur d'un bingo solidaire, d'un mariage ou d'un enterrement se doit de rendre aux personnes l'ayant soutenu, les bénéficiaires des fundraising devront participer aux collectes mises en place par leurs donateurs (collègues, parents, amis). La paroisse " rembourse " elle aussi en partie ses fidèles lorsqu'ils sont dans le besoin. Selon l'enquête quantitative menée en 2002 par Margaret Chung et David Hill, après la famille, l'Eglise représente la seconde source d'aide financière et alimentaire dans les communautés urbaines de Port-Vila et Luganville (2002 : 34). Notons que l'adhésion, principalement féminine, aux associations chrétiennes assure également selon Bronwen Douglas une protection, un espace de respect où les femmes peuvent construire des liens de solidarité et de confiance, développer leurs compétences de leadership ou de gestion (Douglas, $2003: 6,8$ ).

À Seaside Tongoa, les parties de bingos et les fundraising - mais aussi les alliances, les funérailles, les envois interinsulaires d'argent et de biens, etc. - sont apparentés. Chacun d'entre eux constitue des systèmes d'échanges correspondant à différentes formes de sociabilité et mettant en scène différents types d'acteurs (les femmes, les voisins, la famille étendue, etc.). Ces formes d'échange révèlent l'existence de plusieurs com- munautés au sein de Seaside Tongoa ou transcendant cette entité. Les systèmes peuvent être entremêlés. Les parties de bingo sont parfois accompagnées d'une vente de plats 22 ou de kava, tout comme un mariage ou un décès peuvent être précédés d'un fundraising ou de temps de jeu afin de collecter des fonds. D'après Monnerie (2008 : 48), lorsqu'un système d'échanges est organisé pour en financer un second - à Arama (Nouvelle-Calédonie), un marché peut précéder une cérémonie régionale de la Grande Maison il existe une distinction hiérarchique claire entre ces deux formes. Les " complexes d'échanges" (système d'échanges combiné à un mode spécifique de sociabilité et d'usage de la langue) nouvellement créés sont selon lui subordonnés aux « complexes » qu'il nomme " traditionnels ». En adoptant cette interprétation, on pourrait comprendre pourquoi les habitants de Seaside Tongoa ne jouent pas au bingo lors de funérailles ou de mariages et pourquoi il est mal considéré de débuter une partie de bingo le dimanche ${ }^{23}$. Si certains systèmes d'échange sont subordonnés à d'autres, peut-on dire qu'il existe également un ordonnancement hiérarchique règlant les sentiments d'appartenance communautaire ? Un système d'échange participe à l'entretien de différents sentiments communautaires et un sentiment communautaire ne correspond pas à un système d'échange spécifique mais est entretenu de multiples façons. Le sentiment d'appartenir à une communauté donnée est entremêlé à d'autres affiliations et éprouvé en certaines occasions seulement. Il faudrait plus d'enquêtes pour essayer de déterminer si certains sentiments et contextes sont plus valorisés que d'autres.

\section{Vers le « bingo pognon » ?}

\section{Conséquences socioéconomiques}

En utilisant les données disponibles sur la Papouasie Nouvelle-Guinée, Hayano délimite deux formes de jeux d'argent aux extrémités d'un continuum. Â la première extrémité, le jeu de cartes peut être décrit principalement comme une activité récréative ou d'amusement, à l'autre extrémité se trouve le même jeu aux derniers stades d'acculturation et de développement économique qui est joué avec plus d'intensité et a de sérieuses conséquences en dehors de la partie

21. Le livret créé par la Scripture Union Vanuatu remet cependant en cause l'utilisation des jeux d'argent afin de collecter des fonds pour une communauté (Paschke, $2008: 15$ ).

22. Lors de la séance du 23/10/2009, l'organisatrice vend des kumala (patates douces) à 20 vatus $(0,14 €)$ l'unité et du poulet à 50 vatus $(0,34 €)$ la cuisse.

23. Cependant, la transmission de génération en génération du complexe d'échange religieux s'appuie en partie sur des instructions écrites et ne serait pas " traditionnel " d'après la définition qu'en donne Monnerie : "an inherited pattern of thought and action which was handed down from one generation to another without written instruction» (2008: 29). Mais cette définition doit être à interroger. 
(1989 : 239). Ainsi, les jeux d'argent purement récréatifs n'ont pas d'impact sur la situation financière de la personne et ne mettent pas en danger sa sécurité physique (Goffman, 1961, in Hayano, 1989 : 239). Dans leur forme opposée, les jeux d'argent sérieux et institués attirent des " professionnels", dont les seules sources de revenu sont les parties de cartes (Maclean, 1984, in Hayano, 1989 : 239). Au Vanuatu, si les machines à sous des casinos peuvent attirer un certain nombre de professionnels, les parties de bingo de Seaside Tongoa ne concernent pas le même type de joueurs. Sur le continuum proposé par David Hayano, les caractéristiques du jeu d'argent mis en place dans cette communauté du Vanuatu se situent entre celles des jeux récréatifs et celles des jeux institutionnalisés, mais nettement plus proches de celles du premier type. En effet, lorsque les femmes ont autre chose à faire que de jouer aux cartes, comme regarder la télévision ou préparer une cérémonie telle un mariage ou des funérailles, la plupart des groupes arrêtent leur partie. La sécurité physique des joueurs est également assurée. Aucune bagarre n'est déclenchée. De plus, si le bingo solidaire dégage de grosses sommes d'argent, il n'engendre pas réellement d'enrichissement du bénéficiaire parce que celui-ci sera amené à rendre à plus ou moins long terme lorsque ses associés - ou toute autre personne ayant participé à la soirée - organiseront leur propre bingo de collecte de fonds ou un fundraising. De même à Arama (Nouvelle-Calédonie), les gains amassés par les organisateurs lors des bingos des petits ou grands marchés (20 à $50 \%$ des mises) sont redistribués à l'échelle du village ou partagés entre les associations du fait de l'organisation à tour de rôle de ces formes d'échange (Monnerie, 2008 : 40-42). Le continuum d'Hayano prend principalement en considération les répercussions économiques de la pratique des jeux d'argent sans se pencher sur leurs conséquences sociales. La complexité dans laquelle les jeux d'argent prennent place et leur capacité à nourrir ou à affecter les sentiments communautaires ne sont pas prises en compte.

\section{Un même nom pour des réalités différentes}

En 1989, Hayano pense que la monétarisation croissante des économies locales de Papouasie Nouvelle-Guinée va inévitablement transformer les parties de cartes récréatives en un sérieux bisness (1989 : 241). D’après l'article paru dans Les Nouvelles calédoniennes précédemment cité, à Nouméa, du fait de la tolérance policière, le «bingo pognon " est en plein essor. Tout comme Sabourin et Tyuienon (2007 : 316), Monnerie (2008: 40) a mis en évidence, dans la province Nord, des formes de bingo fondées sur la réciprocité et la redistribution. Mais d'après l'article des Nouvelles calédoniennes, en ville " les habitués se retrouvent par centaines tous les jours, parfois jusqu’à 3 heures du matin, et les parties brassent de plus en plus d'argent ». Les organisatrices et les ramasseuses sont des professionnelles qui conservent une partie des mises en tant que salaire. Un bingo officiel, dont la majorité des capitaux sont publics, existe également à Nouméa (Anonyme, 2009). Les bingos solidaires ou passe-temps pratiqués à Seaside Tongoa en 2009 ne sont donc pas du même ordre que le bingo pognon de la capitale kanak. Nous pouvons néanmoins supposer qu'avec le développement économique de Seaside Tongoa, les bingos passetemps et d'entraide diminueront ou disparaîtront au profit des bingos lucratifs et institués. Outre l'essor économique, l'institution d'un système de protection sociale efficace pourrait également conduire à la disparition progressive des bingos de solidarité. En effet, de tels systèmes ont été principalement mis en place afin de lutter contre l'insécurité produite par les aléas de la santé, de l'emploi, des finances, etc. Notons cependant que les débats sociologiques menés sur le rôle de l'Etat providence en France ont mis en avant l'existence de nouvelles formes d'échange intergénérationnel au sein de la famille (gardes d'enfants, aides financières, etc.) ( $c f$. Attias-Donfut, 1995). Lorsqu'un système s'étatise, les solidarités se modifient plutôt qu'elles ne déclinent.

\section{Conclusion}

L'analyse de la pratique du bingo à Seaside Tongoa montre de nombreuses similitudes entre les formes quotidienne et occasionnelle de ce jeu de hasard. Malgré le caractère ludique et lucratif de leur activité, ces deux types de bingo ne répondent ni à la définition du loisir établie par Yonnet (1985: 64), ni à celle du bingo pognon de Nouméa. Le bingo solidaire tout comme le bingo passe-temps ne sont pas détachés des obligations sociales et les reproches faits aux femmes qui ne daignent pas participer aux parties révèlent l'importance relationnelle que revêtent ces activités. Au quotidien, le bingo entretient des relations sociales au sein de petites communautés résidentielles et féminines. Par le partage de temps récréatifs, les femmes nourrissent jour après jour des sentiments communautaires fondés sur le genre et le lieu de résidence. Le bingo passe-temps constitue avant tout un mode de sociabilité. Quant au bingo solidaire, il s'apparente à une collecte d'argent et mobilise les principes de la réciprocité des dons. L'aide économique est perçue à tour de rôle par les participants. Une personne souhaitant organiser un bingo solidaire est ainsi soutenue par ceux qu'elle a précédemment aidés à rassembler des fonds. Le bingo 
occasionnel n'attire pas le même public que les bingos du quotidien : si les femmes sont toujours présentes, les hommes entrent en scène et le réseau social mobilisé dans l'activité s'étend. Lorganisateur du bingo fait appel à ses parents résidant à Seaside Tongoa et dans la capitale ainsi qu'à ses amis et collègues de travail. Des petits groupes de trois ou quatre personnes, au sein desquels une sorte de contrat d'entraide économique est établi, peuvent également se mettre en place. Des liens sociaux solidaires au sein de Seaside Tongoa, du quartier de Seaside et entre les différents groupements de man Tongoa de Port-Vila sont formés - ou du moins entretenus - par l'intermédiaire de ces bingos et des relations de dette qu'ils engendrent. Le bingo solidaire constitue ainsi un système d'échange redistributif nourrissant différentes formes de sociabilité.

Cela dit, malgré la plus grande capacité des bingos de collecte de fonds à entretenir des relations de solidarité, les bingos quotidiens et occasionnels ne s'opposent pas et ne relèvent pas de logiques contradictoires. Ces deux formes de jeu de tirage participent chacune à leur manière à l'entretien de sentiments communautaires multiples qui sont parfois chacun vécus par les individus de façon passagère. Les bingos passetemps et solidaires sont deux nouvelles formes d'échanges redistributifs et de sociabilité à prépondérance féminine, participant au renouvellement et au renforcement des communautés, sans pour autant mettre en danger les systèmes d'échanges plus anciens.

\section{BIBLIOGRAPHIE}

Anonyme, 2009 (28/08). Nouvelle Calédonie : l'envolée des bingos sauvages, Vanuatu Infos, pp. 3, article repris des Nouvelles calédoniennes (24/08/2009).

Attias-Donfut Claudine, 1995. Les solidarités entre les générations. Vieillesse, famille, État, Paris, Nathan, Essais et recherche.

Becker Howard S., 1985. Outsiders, Études de sociologie de la déviance: fumeurs de marijuana, musiciens de jazz, entrepreneurs de morale, policiers et délinquants, Paris, Éditions Métailié, Observations.

BEDFORD Richard, 1973. A transition in circular mobility, population movement in the New Hebrides, 1800-1970, in H. Brookfield (ed.), The Pacific in transition, geographical perspectives on adaptation and change, Canberra, Australian National University Press, pp. 187-228.
Bonnemaison Joël, 1970. Note préliminaire sur les terroirs et structures agraires des iles du Centre et du Nord des Nouvelles-Hébrides, Compte-rendu de missions : années 1969-1970, Nouméa, ORSTOM.

-, 1977. Systeme de migration et croissance urbaine à Port-Vila et Luganville (Nouvelles-Hébrides), Paris, orstom, Travaux et documents de l'orstom 60.

Charpentier Jean-Michel, 1998. Identité et communication au Vanuatu, in D. Tryon and P. de Deckker (éds), Identités en mutation dans le Pacifique à l'aube du troisième millénaire. Hommage à Joël Bonnemaison (1940-1997), actes du colloque tenu à l'ambassade d'Australie (Paris, 29-30 mai 1997), Bordeaux, Presses universitaires de Bordeaux, îles et $\mathrm{Ar}$ chipels 26, pp. 85-100.

Chung Margaret and David HiLl, 2002. Urban informal settlements in Vanuatu: Challenge for equitable development, rapport pour le Pacific Islands Forum Secretariat et l'Un Economic and social commission for Asia and the Pacific, Pacific Operation Centre.

Douglas Bronwen, 2003. Christianity, tradition, and everyday modernity: towards an anatomy of women's groupings in Melanesia, Oceania 74 (1/2), pp. 6-23.

Durкheim Émile, 2007 [1897]. De la division du travail social, Paris, Presses universitaires de France.

—, 1975. Textes. 1. Eléments d'une théorie sociale, Paris, Éditions de Minuit, Le sens commun.

ERIKSEn Annelin, 2008. Gender, christianity and change in Vanuatu: An analysis of social movements in North Ambrym, Ashgate, Anthropology and Cultural History in Asia and the Indo-Pacific.

Goodale Jane, 1987. Gambling is hard work: Card playing in Tiwi society, Oceania 58 (1), pp. 6-21.

Hayano David M., 1989. Like eating money: card gambling in a Papua New Guinea Highlands village, Journal of gambling behavior 5 (3), pp. 231-245.

Kelly Susanna Katharine, 1999. Unwrapping mats: People, land and material culture in Tongoa, Central Vanuatu, Ph.D. Thesis, University College London.

Législation De la république du Vanuatu, 2006. Chapitre 10 : Jeux de hasard (interdiction).

LeIF Joseph, 1944. Introduction du traducteur, in F. Tönnies, Communauté et société. Catégo- 
ries fondamentales de la sociologie pure, Paris, Presses universitaires de France, pp. v-XXXI.

Lightner Sara and Anna Naupa, 2005. Histri blong yumi long Vanuatu: an educational resource: teacher's guide, vol. 3, Port Vila, Vanuatu, Vanuatu Cultural Centre.

Mauss Marcel, 1950. Sociologie et Anthropologie, Paris, Presses universitaires de France.

Mitchell William, 1988. The defeat of hierarchy: gambling as exchange in a Sepik society, American ethnologist 15 (4), pp. 638-657.

Monnerie Denis, 2005. La parole de notre Maison. Discours et cérémonies kanak aujourd'hui, Paris, CNRs Éditions et Éditions de la Maison des sciences de l'homme.

—, 2008. The Great House and the Marché. Two Kanak exchange complexes (New-Caledonia), in P. Stewart and A. Strathern (eds), Exchange and Sacrifice, Durham, North Carolina, Carolina Academic Press, pp. 27-54.

PAкоA George, 1987. Sheperd islands, extended family system, Port-Vila, Vanuatu.

Paschke Jon, 2008. Gambling mo yu, Port-Vila, Scripture Union Vanuatu.

Rubinstein Robert, 1987. The changing context of card playing on Malo, Vanuatu, Oceania 58 (1), pp. 47-59.
Sabourin Éric et Raymond Tyuienon, 2007. Produits, monnaie et bingo : les marchés ruraux en Nouvelle-Calédonie entre échange et réciprocité, Revue du MAUSS 29 (1), pp. 301327.

SeXton Lorraine, 1987. The social construction of card playing among the Daulo, Oceania 58 (1), pp. 38-46.

Tabani Marc Kurt, 2002. Les pouvoirs de la coutume à Vanuatu. Traditionalisme et édification nationale, Paris, L'Harmattan, Connaissance des hommes.

Weber Max, 1995. Économie et société 2 : L'organisation et les puissances de la société dans leur rapport avec l'économie, Paris, Plon, Agora.

Yonnet Paul, 1985. Jeux, modes et masses : La société française et le moderne 1945-1985, Paris, Gallimard, Bibliothèque des Sciences humaines.

Zimmer Laura, 1986. Card playing among the Gende: A system for keeping money and social relationships alive, Oceania 56 (4), pp. 245-263.

—, 1987. Playing at being men, Oceania 58 (1), pp. 22-37. 